\title{
MicroRNA-503-5p improves carotid artery stenosis by inhibiting the proliferation of vascular smooth muscle cells
}

\author{
ZHIYUAN YAN ${ }^{1}$, HONG WANG $^{1}$, JUNJUN LIANG ${ }^{2}$, YUAN LI $^{3}$ and XIAODONG LI ${ }^{1}$ \\ Departments of ${ }^{1}$ Neurology, ${ }^{2}$ Neurosurgery and ${ }^{3}$ Electrocardiography, \\ Anqiu People's Hospital, Weifang, Shandong 262100, P.R. China
}

Received November 1, 2019; Accepted February 11, 2020

DOI: $10.3892 /$ etm.2020.9213

\begin{abstract}
Carotid artery stenosis (CAS) is a common arteriosclerotic vascular disease affected by vascular smooth muscle cells (VSMCs). The aim of the present study was to investigate the expression and diagnostic value of microRNA (miR)-503-5p in asymptomatic patients with CAS and to further explore the effect of miR-503-5p on VSMC proliferation. The levels of miR-503-5p in the serum of 62 asymptomatic patients with CAS and 60 healthy controls were detected by reverse transcription-quantitative PCR. The association between miR-503-5p and the clinical characteristics of the patients was analyzed using the $\chi^{2}$ test. A receiver operating characteristic curve was drawn to evaluate the diagnostic value of miR-503-5p to distinguish asymptomatic patients with CAS from healthy controls. Finally, miR-503-5p inhibitors and mimics were transfected into VSMCs in vitro to detect the effect of miR-503-5p on the proliferation ability through Cell Counting Kit- 8 assays. The serum levels of miR-503-5p in asymptomatic patients with CAS were significantly reduced as compared with those in healthy individuals. The expression levels of miR-503-5p were significantly associated with diabetes and arterial stenosis. Furthermore, the area under the ROC curve was 0.817 , the specificity was $79.03 \%$ and the sensitivity was $83.30 \%$, which proved that miR-503-5p had a high diagnostic accuracy in patients with CAS. Finally, the in vitro proliferation assay indicated that overexpression of miR-503-5p significantly inhibited the proliferation of VSMCs. In conclusion, miR-503-5p is a potential diagnostic biomarker for asymptomatic CAS and overexpression of miR-503-5p may inhibit the proliferation of VSMCs and improve CAS.
\end{abstract}

Correspondence to: $\mathrm{Dr}$ Xiaodong $\mathrm{Li}$, Department of Neurology, Anqiu People's Hospital, 159 Xuefu Road, Weifang, Shandong 262100, P.R. China

E-mail: 1xd860290@163.com

Key words: microRNA-503-5p, asymptomatic carotid artery stenosis, diagnostic, vascular smooth muscle cells

\section{Introduction}

Carotid artery stenosis (CAS) is an important risk factor for ischemic neurological events. Nearly $80 \%$ of strokes occur in asymptomatic patients and the incidence increases with age $(1,2)$. In addition, a number of cases were identified to suffer from coronary artery disease or peripheral atherosclerosis in asymptomatic patients with CAS (3). CAS is initially caused by abnormal proliferation of vascular smooth muscle cells (VSMCs), accompanied by intimal hyperplasia caused by matrix deposition of extracellular connective tissue to form plaques and eventually develops into symptomatic stenosis $(4,5)$. There are various measures to prevent or treat CAS, including endarterectomy, endovascular stent placement (6) and medication (7). However, prophylactic surgery for CAS is controversial for patients with asymptomatic CAS (8). Statins and anti-platelet drugs are associated with a certain degree of stroke risk (9). Therefore, it is necessary to search for novel subclinical molecular biomarkers to reliably predict whether asymptomatic CAS is likely to develop into symptomatic CAS or remain stable.

MicroRNAs (miRNAs) are a group of highly conserved small non-coding RNA molecules that act as negative regulators of post-transcriptional regulation by inhibiting target gene expression. miRNAs have been indicated to be abnormally expressed in the physiological and pathological processes of various diseases. Numerous miRNAs have been reported to be abnormally expressed in CAS, including miR-330-5p (10) and miR-125a-3p (11), indicating that miRNAs have potential regulatory effects in CAS. In addition, certain miRNAs are considered potential markers of atherosclerosis and miRNAs also have an important role in VSMCs. Bi et al (12) reported that miR-503 inhibits the proliferation and migration of human aortic VSMCs induced by platelet-derived growth factors via targeting insulin receptors. Furthermore, Cremer et al (13) recently reported that miR-503-5p was associated with MALAT1 to reduce atherosclerosis in mice. In addition, miR-503-5p may regulate the differentiation of the mesenchymal stem cells into VSMCs, which has a certain effect in vascular tissue transplantation (14). However, the diagnostic value of miR-503-5p in CAS and the effect of miR-503-5p on the proliferation of VSMCs in CAS remains to be determined.

In the present study, the diagnostic value of miR-503-5p in patients with CAS was evaluated by detecting the expression 
levels of miR-503-5p in patients with CAS. The effect of miR-503-5p on the proliferation of VSMCs in CAS was further investigated.

\section{Materials and methods}

Patient recruitment and sample collection. A total of 62 asymptomatic patients with CAS encountered at Anqiu People's Hospital (Weifang, China) between February 2010 and February 2014 were included. The selection criteria were an age of $\geq 18$ years and asymptomatic CAS identified based on the patients' clinical data and the National Institutes of Health Stroke Scale (15). Exclusion criteria were a history of stroke, transient ischemic attack, coronary instability, congestive heart failure, chronic or acute inflammatory conditions, cancer and recent intracranial hemorrhage. From the health check-up center, 60 healthy controls with a similar age were selected as the control group. The inclusion criteria were no history of stroke and no mental illness. The patients' basic data and clinical characteristics were recorded accordingly (Table SI) and blood samples were obtained on the day of hospitalization. After taking blood samples, the serum samples were collected by centrifugation at $2,500 \mathrm{x}$ for $15 \mathrm{~min}$ at room temperature in a swinging-bucket centrifuge (Centrifuge 5810R; Eppendorf) and stored at $-80^{\circ} \mathrm{C}$.

Cell culture and transfection. Human (h)VSMCs were purchased from the American Type Culture Collection and cultured in Dulbecco's modified Eagle's medium (Gibco; Thermo Fisher Scientific, Inc.) with $10 \%$ fetal bovine serum (Thermo Fisher Scientific, Inc.). The cells were cultured in an incubator at $37^{\circ} \mathrm{C}$ with $5 \% \mathrm{CO}_{2}$. Cell transfection was performed when the cells were $\sim 60 \%$ confluent. Transfected vectors were miR-503-5p mimics (cat. no. miR10002874-1-5; Guangzhou RiboBio Co., Ltd.), miR-503-5p inhibitor (cat. no. miR20002874-1-5; Guangzhou RiboBio Co., Ltd.), mimics negative control (mimics NC; cat. no. miR1N0000001-1-5; Guangzhou RiboBio Co., Ltd.) and inhibitor NC (cat. no. miR2N0000001-1-5; Guangzhou RiboBio Co., Ltd.), respectively. The transfection reagent was Lipofectamine ${ }^{\circledR} 2000$ (Invitrogen; Thermo Fisher Scientific, Inc.).

$R N A$ extraction and reverse transcription-quantitative $P C R$ (RT- $q P C R)$. TRIzol was used to extract total RNA from the subjects' serum and miRNA was isolated using the miRNApure Mini Kit (CWBiotech). The extracted RNA was reverse-transcribed into complementary (c)DNA according to the specifications of the Super cDNA First-Strand Synthesis Kit (CWBiotech). Finally, qPCR was performed with the Ultra SYBR Mixture and the ROX Assay kit (CWBiotech) in an ABI 7300 real-time PCR system (Applied Biosystems; Thermo Fisher Scientific, Inc.). The reaction mixtures were incubated at $95^{\circ} \mathrm{C}$ for $10 \mathrm{~min}$, followed by 40 cycles of $94^{\circ} \mathrm{C}$ for $15 \mathrm{sec}$, $55^{\circ} \mathrm{C}$ for $30 \mathrm{sec}$ and $70^{\circ} \mathrm{C}$ for $30 \mathrm{sec}$. Primer sequences used are as following: miR-503-5p forward 5'-CCTATTTCCCAT GATTCCTTCATA-3' and reverse 5'-CTCGTTCGGCAGCAC A-3'; and U6 forward 5'-AACGCTTCACGAATTTGCGT-3' and reverse 5'-CTCGTTCGGCAGCACA-3'. Using U6 RNA as the internal control, the relative expression of miR-503-5p was calculated using the $2^{-\Delta \Delta C q}$ method (16). All of the experiments were repeated independently three times.
Cell proliferation assay. VSMCs at the exponential growth phase after transfection were seeded at $5 \times 10^{4}$ cells per well in 96-well plates for continuous culture for 3 days and their proliferation capacity was determined at $0,24,48$ or $72 \mathrm{~h}$. Each time-point was performed in triplicate. Prior to detection, $10 \mu \mathrm{l}$ Cell Counting kit 8 (CCK-8) reagent was added to each well, followed by further incubation for $1 \mathrm{~h}$. The absorbance value was then detected at $490 \mathrm{~nm}$ using a Bio-Rad iMark plate reader (Bio-Rad Laboratories, Inc.). The effect of miR-503-5p on the proliferation ability of VSMCs was evaluated after 3 days of continuous detection.

Statistical analysis. All statistical data were processed and analyzed with SPSS 21.0 software (IBM Corp.) and GraphPad Prism 7.0 software. Student's t-test and one-way analysis of variance followed by Tukey's test were used to detect differences between groups. The $\chi^{2}$ test was used to analyze the association between miR-503-5p expression and clinical characteristics of patients. A receiver operating characteristic (ROC) curve was drawn to evaluate the diagnostic value of miR-503-5p in CAS and calculate the area under the curve (AUC). $\mathrm{P}<0.05$ was considered to indicate a statistically significant difference.

\section{Results}

Serum levels of miR-503-5p in asymptomatic patients with $C A S$. The levels of miR-503-5p in the serum of all subjects were detected by RT-qPCR. The results indicated that the levels of miR-503-5p in asymptomatic patients with CAS was significantly lower than those in healthy controls $(\mathrm{P}<0.001$; Fig. 1), suggesting that miR-503-5p may have a critical role in the development of asymptomatic CAS.

Association between miR-503-5p and clinicopathological features of patients. The association between the expression levels of miR-503-5p and the clinicopathological features of asymptomatic patients with CAS was then explored. According to the average expression levels of miR-503-5p in asymptomatic patients with CAS, all patients with asymptomatic CAS were divided into the high miR-503-5p expression group $(n=24)$ and the low miR-503-5p expression group $(n=38)$. As presented in Table I, the expression levels of miR-503-5p were not significantly associated with age, gender, body mass index (BMI), hypertension and dyslipidemia $(\mathrm{P}>0.05)$, but were significantly associated with diabetes $(\mathrm{P}=0.034)$ and carotid artery stenosis $(\mathrm{P}=0.017)$.

Diagnostic value of miR-503-5p in asymptomatic patients with CAS. A ROC curve was drawn according to the expression levels of miR-503-5p in asymptomatic patients with CAS and healthy controls to evaluate the diagnostic value of miR-503-5p for CAS. As indicated in Fig. 2, the AUC of the ROC curve was 0.817 . The sensitivity was $83.30 \%$, the specificity was $79.03 \%$ and the cut-off value was 0.810 . The results suggested that miR-503-5p was of high diagnostic value in asymptomatic patients with CAS.

miR-503-5p regulates the proliferation of hVSMCs. The occurrence of CAS is linked to the abnormal proliferation 


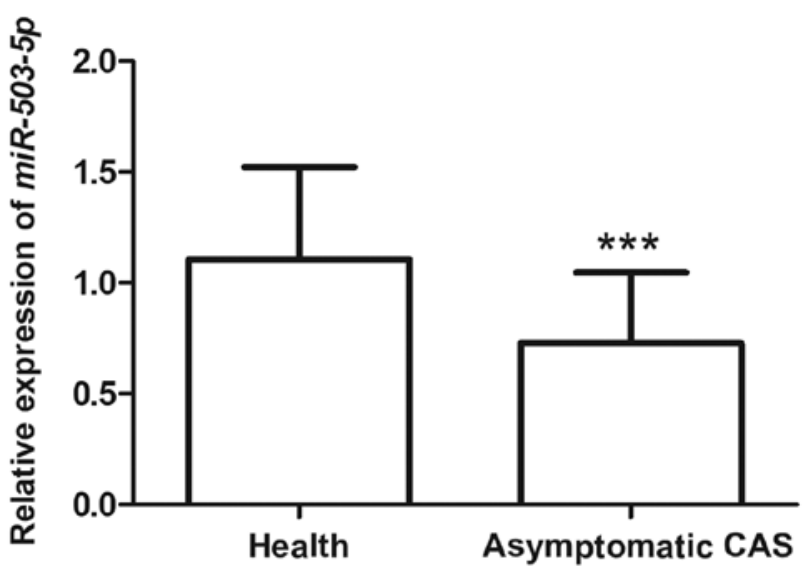

Figure 1. Expression of miR-503-5p in the serum of asymptomatic patients with CAS and the healthy control group detected by reverse transcription-quantitative PCR. Compared with that in the healthy control group, the expression of miR-503-5p in the serum of asymptomatic patients with CAS was significantly decreased. ${ }^{* * *} \mathrm{P}<0.001$. CAS, carotid artery stenosis; miR, microRNA.

of VSMCs. In the present study, the effect of miR-503-5p on the proliferation of hVSMCs was verified by transfection of miR-503-5p mimics and inhibitors. The transfection efficiency was verified by RT-qPCR analysis. As presented in Fig. 3A, the expression in the miR-503-5p mimics group was significantly higher than that in the control group $(\mathrm{P}<0.001)$, while the expression in the miR-503-5p inhibitor group was significantly lower $(\mathrm{P}<0.001)$. The results suggested that the overexpression and knockdown efficiency of miR-503-5p mimics and inhibitors in hVSMCs was higher.

A CCK-8 assay was used to further detect the effect of miR-503-5p on the proliferation ability of hVSMCs. As presented in Fig. 3B, the proliferation ability in the miR-503-5p mimics group was lower than that in the control group, while the proliferation ability in the miR-503-5p inhibitor group was significantly higher than that in the control group $(\mathrm{P}<0.01)$.

\section{Discussion}

The carotid artery is the major blood vessel in the neck that supplies blood from the heart to the brain and face. CAS is a pathological condition of vascular stenosis caused by atherosclerotic plaque (17). Asymptomatic CAS is defined as a patient without a history of ischemic stroke or transient ischemic attack in the ipsilateral carotid region and without focal neurological symptoms (18). At present, CAS is diagnosed by double ultrasound, CT angiography and MR angiography (19). Although traditional digital subtraction angiography is the gold standard for CAS diagnosis, its invasive nature carries the risk of stroke. Duplex ultrasound is non-radiative and non-invasive, but its sensitivity and specificity in diagnosing CAS are only moderate and require secondary verification $(20,21)$. Therefore, it is urgent to develop novel sensitive, specific and non-invasive diagnostic markers for CAS.

In recent years, due to its high sensitivity, specificity and non-invasive nature, the detection of miRNAs as biomarkers has attracted an increasing amount of attention (22). In human
Table I. Association of miR-503-5p with the clinical parameters in asymptomatic patients with CAS $(n=62)$.

\begin{tabular}{|c|c|c|c|c|}
\hline \multirow[b]{2}{*}{ Parameter } & \multirow[b]{2}{*}{ Total } & \multicolumn{2}{|c|}{$\begin{array}{c}\text { miR-503-5p } \\
\text { expression }\end{array}$} & \multirow[b]{2}{*}{ P-value } \\
\hline & & $\begin{array}{c}\text { Low } \\
(n=38)\end{array}$ & $\begin{array}{l}\text { High } \\
(n=24)\end{array}$ & \\
\hline Age, years & & & & 0.586 \\
\hline$\leq 58$ & 22 & 15 & 7 & \\
\hline$>58$ & 40 & 23 & 17 & \\
\hline Gender & & & & 0.792 \\
\hline Male & 36 & 23 & 13 & \\
\hline Female & 26 & 15 & 11 & \\
\hline BMI & & & & 0.444 \\
\hline$\leq 25$ & 32 & 18 & 14 & \\
\hline$>25$ & 30 & 20 & 10 & \\
\hline Dyslipidemia & & & & 0.068 \\
\hline No & 29 & 14 & 15 & \\
\hline Yes & 33 & 24 & 9 & \\
\hline Hypertension & & & & 0.063 \\
\hline No & 26 & 12 & 14 & \\
\hline Yes & 36 & 26 & 10 & \\
\hline Diabetes & & & & 0.034 \\
\hline No & 23 & 10 & 13 & \\
\hline Yes & 39 & 28 & 11 & \\
\hline Degree of CAS, \% & & & & 0.017 \\
\hline $50-69$ & 26 & 11 & 15 & \\
\hline $70-99$ & 36 & 27 & 9 & \\
\hline
\end{tabular}

miR, microRNA; BMI, body mass index; CAS, carotid artery stenosis.

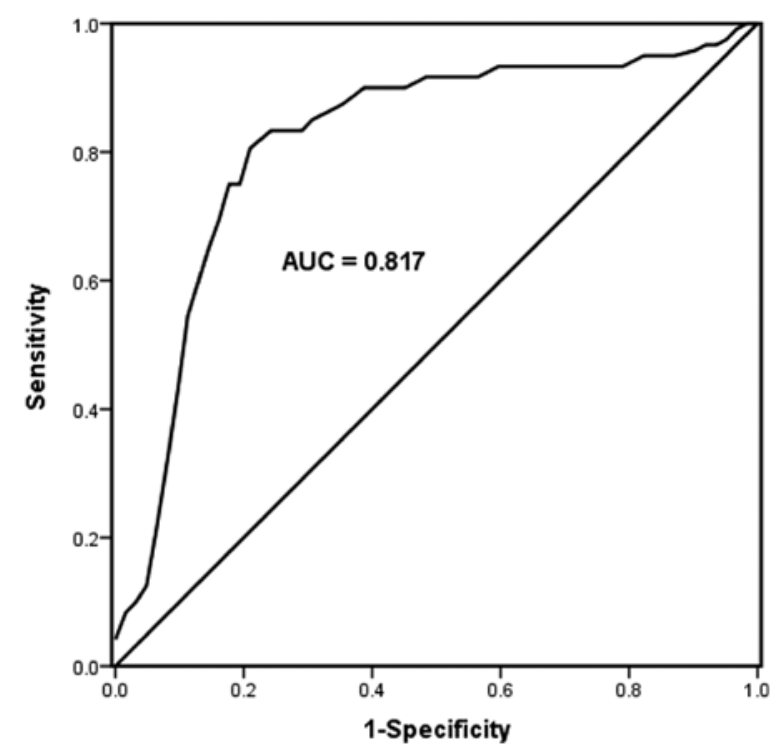

Figure 2. ROC curve for evaluating the diagnostic value of miR-503-5p for asymptomatic patients with carotid artery stenosis. The AUC was 0.826 , the sensitivity was $85.30 \%$, the specificity was $79.03 \%$ and the cut-off value was 0.826 . AUC, area under the ROC curve; ROC, receiver operating characteristic. 

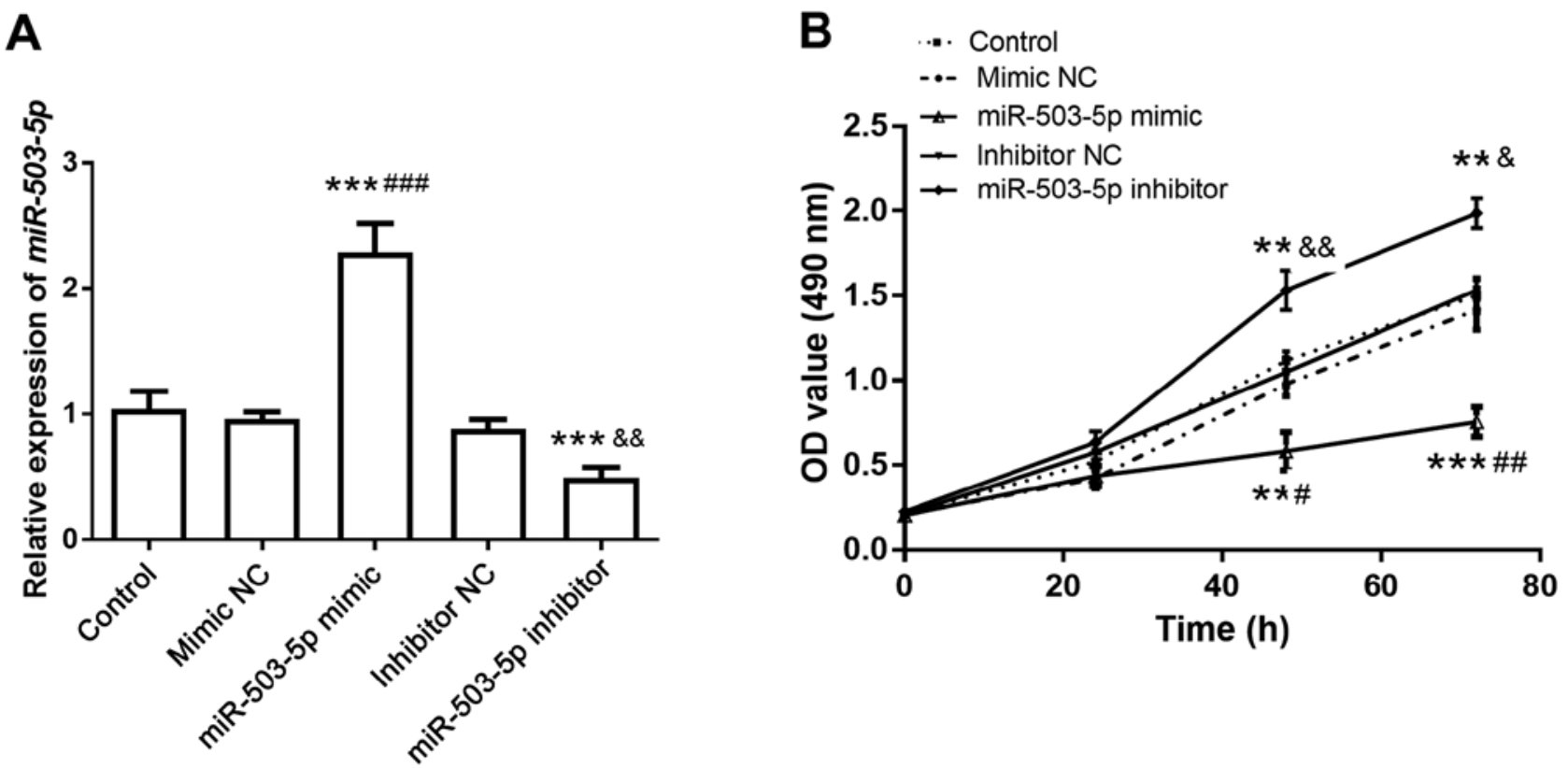

Figure 3. Detection of cell proliferation after transfection with miR-503-5p inhibitors and mimics. (A) The expression levels of miR-503-5p changed after transfection of miR-503-5p inhibitor and mimics, respectively. (B) After transfection with miR-503-5p inhibitors and mimics, the proliferation ability of cells was detected with a Cell Counting Kit-8. After transfection with miR-503-5p inhibitor, the proliferative ability of the cells was significantly promoted, whereas after transfection with miR-503-5p mimics, the proliferation ability was significantly decreased. ${ }^{* *} \mathrm{P}<0.01,{ }^{* * * *} \mathrm{P}<0.001$, compared with control group; ${ }^{\sharp} \mathrm{P}<0.05$, ${ }^{\# \#} \mathrm{P}<0.01,{ }^{\# \#} \mathrm{P}<0.001$, compared with mimic NC group; ${ }^{\circledR} \mathrm{P}<0.05$, \&\& $\mathrm{P}<0.01$, compared with inhibitor NC group. miR, microRNA; NC, negative control; OD, optical density.

cancer, certain serum miRNAs may be used as diagnostic and prognostic markers, including miR-1290 (23), miR-141 (24) and miR-193b (25). Disorder of miRNAs is also involved in the pathological processes of numerous cardiovascular diseases, including atherosclerosis and CAS. For instance, inhibition of miR-103 may reduce the inflammatory response and endoplasmic reticulum stress in patients with atherosclerosis (10). Serum miR-638 was significantly reduced in high-risk CAS patients undergoing carotid endarterectomy and was indicated to serve as a non-invasive biomarker associated with plaque vulnerability and ischemic stroke (26). In the present study, the expression of miR-503-5p was significantly downregulated in asymptomatic patients with CAS compared with that in healthy controls. This confirms that miR-503-5p has a crucial role in patients with CAS. miR-503 has been previously reported to be significantly downregulated in the serum of patients with coronary heart disease and to be a good prognostic marker of coronary heart disease (27). Ezetimibe inhibits PMA-induced monocyte/macrophage differentiation by upregulating the expression of miRNAs, including miR-503, and exerts an anti-atherosclerosis effect (28). These studies are consistent with the results of the present study.

To further confirm the potential role of miR-503-5p in asymptomatic CAS, its diagnostic value was examined. The experimental results indicate that miR-503-5p is highly specific and sensitive in distinguishing asymptomatic patients with CAS from healthy individuals. The clinical value of miR-503-5p has been widely reported in previous studies. Circular RNA (Circrna) 0000267 promotes gastric cancer progression through sponging miR-503-5p and regulation of high-mobility group AT-hook 2 expression (29). Low expression of miR-503 in gastric cancer tissues and serum may be used as a diagnostic marker for gastric cancer (30). In the present study, the promising diagnostic value of miR-503-5p in asymptomatic CAS patients was confirmed. Previous studies have reported that hypertension, dyslipidemia and diabetes are risk factors for the high prevalence of CAS (19). In addition, in patients with type II diabetes, hypertension and dyslipidemia have been indicated to have an accumulating effect on the carotid plaque burden (31). Recently, it has been reported that miR-503-5p is dysregulated in diabetic nephropathy (32). Therefore, in the present study, the clinicopathological features of patients with asymptomatic CAS and the expression of miR-503-5p were examined and it was indicated that the expression levels of miR-503-5p were associated with diabetes and carotid stenosis.

Studies have reported that plaque stability during the formation of CAS is associated with the function of VSMCs and endothelial cells $(33,34)$. Furthermore, miR-145 was indicated to have a key role in CAS by regulating the function of VSMCs (35). At the same time, miR-503 inhibits the proliferation and migration of human aortic VSMCs induced by platelet-derived growth factors by targeting insulin receptors (12). Therefore, in the present study, miR-503-5p inhibitors and mimics were transfected into hVSMCs. After confirming the sufficient overexpression and knockdown efficiency, the proliferation ability of miR-503-5p on VSMCs was further tested. The results suggested that inhibition of miR-503-5p expression significantly promoted the proliferation of VSMCs. Studies have reported that fibroblast growth factor (FGF) expressed in $68 \%$ of cases of CAS (36). Circrna WD repeat domain 77 targets FGF-2 and regulates the proliferation and migration of VSMCs by stimulating miR-124 (37). At the same time, it has been reported that miR-503 inhibits tumor 
angiogenesis and growth by targeting FGF2 and VEGFA (38). In pulmonary hypertension, miR-424- and miR-503-mediated endothelial cell apelin-FGF connections are destroyed (39). In addition, the relevant target of miR-503-5p was detected by TargetScan and it was indicated that epidermal growth factor 2 was the target of miR-503-5p. Therefore, it was speculated that miR-503-5p may regulate the functions of CAS and VSMCs by targeting FGF2. However, how miR-503-5p exerts its role in CAS remains to be further determined.

In conclusion, a series of experimental results have confirmed the low expression of miR-503-5p in asymptomatic patients with CAS and the low expression of miR-503-5p may be used as a potential diagnostic marker of asymptomatic CAS. At the same time, inhibition of miR-503-5p may promote the proliferation of VSMCs and may be used as a potential therapeutic target for CAS.

\section{Acknowledgements}

Not applicable.

\section{Funding}

No funding was received.

\section{Availability of data and materials}

All data generated or analyzed during this study are included in this published article.

\section{Authors' contributions}

ZY, HW, JL and XL designed the study. ZY, HW, JL and YL performed the experiments and interpreted the data. ZY, HW and YL drafted the manuscript. JL and XL revised it critically for important intellectual content. All authors read and approved the final manuscript.

\section{Ethics approval and consent to participate}

The experiment was approved by the Anqiu People's Hospital Ethics Committee (Weifang, China) and written informed consent was obtained from all subjects.

\section{Patient consent for publication}

Not applicable.

\section{Competing interests}

The authors declare that they have no competing interests.

\section{References}

1. Bogousslavsky J, Van Melle G and Regli F: The lausanne stroke registry: Analysis of 1,000 consecutive patients with first stroke. Stroke 19: 1083-1092, 1988.

2. Paciaroni M, Silvestrelli G, Caso V, Corea F, Venti M, Milia P, Tambasco N, Parnetti L and Gallai V: Neurovascular territory involved in different etiological subtypes of ischemic stroke in the perugia stroke registry. Eur J Neurol 10: 361-365, 2003.
3. Taussky P, Hanel RA and Meyer FB: Clinical considerations in the management of asymptomatic carotid artery stenosis. Neurosurg Focus 31: E7, 2011.

4. Davies MG and Hagen PO: Pathobiology of intimal hyperplasia. Br J Surg 81: 1254-1269, 1994.

5. Subbotin VM: Analysis of arterial intimal hyperplasia: Review and hypothesis. Theor Biol Med Model 4: 41, 2007.

6. O'Brien M and Chandra A: Carotid revascularization: Risks and benefits. Vasc Health Risk Manag 10: 403-416, 2014.

7. Bae C, Szuchmacher M and Chang JB: Comparative review of the treatment methodologies of carotid stenosis. Int J Angiol 24: 215-222, 2015.

8. ACST-2 Collaborative Group; Halliday A, Bulbulia R, Gray W, Naughten A, den Hartog A, Delmestri A, Wallis C, le Conte S and Macdonald S: Status update and interim results from the asymptomatic carotid surgery trial-2 (ACST-2). Eur J Vasc Endovasc Surg 46: 510-518, 2013.

9. Abbott AL: Medical (nonsurgical) intervention alone is now best for prevention of stroke associated with asymptomatic severe carotid stenosis: Results of a systematic review and analysis. Stroke 40: e573-e583, 2009.

10. Wei X, Sun Y, Han T, Zhu J, Xie Y, Wang S, Wu Y, Fan Y, Sun X, Zhou J, et al: Upregulation of miR-330-5p is associated with carotid plaque's stability by targeting Talin-1 in symptomatic carotid stenosis patients. BMC Cardiovasc Disord 19: 149, 2019.

11. Hu W, Chang G, Zhang M, Li Y, Yin L, Huang Y, Feng C, Gu Y, Wen D and Wang S: MicroRNA-125a-3p affects smooth muscle cell function in vascular stenosis. J Mol Cell Cardiol 136: 85-94, 2019.

12. Bi R, Ding F, He Y, Jiang L, Jiang Z, Mei J and Liu H: miR-503 inhibits platelet-derived growth factor-induced human aortic vascular smooth muscle cell proliferation and migration through targeting the insulin receptor. Biomed Pharmacother 84: 1711-1716, 2016.

13. Cremer S, Michalik KM, Fischer A, Pfisterer L, Jaé N, Winter C, Boon RA, Muhly-Reinholz M, John D, Uchida S, et al: Hematopoietic deficiency of the long noncoding RNA MALAT1 promotes atherosclerosis and plaque inflammation. Circulation 139: 1320-1334, 2019.

14. Gu W, Hong X,Le Bras A, Nowak WN, Issa Bhaloo S, Deng J, Xie Y, $\mathrm{Hu}$ Y, Ruan XZ and Xu Q: Smooth muscle cells differentiated from mesenchymal stem cells are regulated by microRNAs and suitable for vascular tissue grafts. J Biol Chem 293: 8089-8102, 2018.

15. Lal BK, Dux MC, Sikdar S, Goldstein C, Khan AA, Yokemick J and Zhao L: Asymptomatic carotid stenosis is associated with cognitive impairment. J Vasc Surg 66: 1083-1092, 2017.

16. Rao X, Huang $X$, Zhou Z and Lin X: An improvement of the $2^{\wedge}$ (-delta delta CT) method for quantitative real-time polymerase chain reaction data analysis. Biostat Bioinforma Biomath 3: 71-85, 2013.

17. Beckman JA: Management of asymptomatic internal carotid artery stenosis. JAMA 310: 1612-1618, 2013.

18. Goldstein LB: Screening for asymptomatic carotid artery stenosis: Evidence-based opinion. JAMA Intern Med 176: 633-634, 2016.

19. Dharmakidari S, Bhattacharya $P$ and Chaturvedi S: Carotid artery stenosis: Medical therapy, surgery, and stenting. Curr Neurol Neurosci Rep 17: 77, 2017.

20. Nederkoorn PJ, van der Graaf Y and Hunink MG: Duplex ultrasound and magnetic resonance angiography compared with digital subtraction angiography in carotid artery stenosis: A systematic review. Stroke 34: 1324-1332, 2003.

21. Jahromi AS, Cina CS, Liu Y and Clase CM: Sensitivity and specificity of color duplex ultrasound measurement in the estimation of internal carotid artery stenosis: A systematic review and meta-analysis. J Vasc Surg 41: 962-972, 2005.

22. Shi HB, Yu JX, Yu JX, Feng Z, Zhang C, Li GY, Zhao RN and Yang XB: Diagnostic significance of microRNAs as novel biomarkers for bladder cancer: A meta-analysis of ten articles. World J Surg Oncol 15: 147, 2017.

23. Sun H, Wang L, Zhao Q and Dai J: Diagnostic and prognostic value of serum miRNA-1290 in human esophageal squamous cell carcinoma. Cancer Biomark 25: 381-387, 2019.

24. Li Z, Ma YY, Wang J, Zeng XF, Li R, Kang W and Hao XK: Exosomal microRNA-141 is upregulated in the serum of prostate cancer patients. Onco Targets Ther 9: 139-148, 2015.

25. Chan CM, Lai KKY, Ng EKO, Kiang MN, Kwok TWH, Wang HK, Chan KW, Law TT, Tong DK, Chan KT, et al: Serum microRNA-193b as a promising biomarker for prediction of chemoradiation sensitivity in esophageal squamous cell carcinoma patients. Oncol Lett 15: 3273-3280, 2018. 
26. Luque A, Farwati A, Krupinski J and Aran JM: Association between low levels of serum miR-638 and atherosclerotic plaque vulnerability in patients with high-grade carotid stenosis. J Neurosurg 131: 72-79, 2018.

27. Fei Y, Hou J, Xuan W, Zhang C and Meng X: The relationship of plasma miR-503 and coronary collateral circulation in patients with coronary artery disease. Life Sci 207: 145-151, 2018.

28. Muñoz-Pacheco P, Ortega-Hernández A, Miana M, Cachofeiro V, Fernández-Cruz A and Gómez-Garre D: Ezetimibe inhibits PMA-induced monocyte/macrophage differentiation by altering microRNA expression: A novel anti-atherosclerotic mechanism. Pharmacol Res 66: 536-543, 2012.

29. Cai X, Nie J, Chen L and Yu F: Circ 0000267 promotes gastric cancer progression via sponging MiR-503-5p and regulating HMGA2 expression. Mol Genet Genomic Med 8: e1093, 2020.

30. Wu D, Cao G, Huang Z, Jin K, Hu H, Yu J and Zeng Y: Decreased miR-503 expression in gastric cancer is inversely correlated with serum carcinoembryonic antigen and acts as a potential prognostic and diagnostic biomarker. Onco Targets Ther 10: 129-135, 2016.

31. Yuan C, Lai CW, Chan LW, Chow M, Law HK and Ying M: Cumulative effects of hypertension, dyslipidemia, and chronic kidney disease on carotid atherosclerosis in Chinese patients with type 2 diabetes mellitus. J Diabetes Res 2014: 179686, 2014.

32. Assmann TS, Recamonde-Mendoza M, Costa AR, Puñales M, Tschiedel B, Canani LH, Bauer AC and Crispim D: Circulating miRNAs in diabetic kidney disease: Case-control study and in silico analyses. Acta Diabetol 56: 55-65, 2019.

33. Chen YC, Bui AV, Diesch J, Manasseh R, Hausding C, Rivera J, Haviv I, Agrotis A, Htun NM, Jowett J, et al: A novel mouse model of atherosclerotic plaque instability for drug testing and mechanistic/therapeutic discoveries using gene and microRNA expression profiling. Circ Res 113: 252-265, 2013.
34. Borissoff JI, Heeneman S, Kilinc E, Kassák P, Van Oerle R, Winckers K, Govers-Riemslag JW, Hamulyák K, Hackeng TM, Daemen MJ, et al: Early atherosclerosis exhibits an enhanced procoagulant state. Circulation 122: 821-830, 2010.

35. Han Z, Hu H, Yin M, Li X, Li J, Liu L and Liu B: miR-145 is critical for modulation of vascular smooth muscle cell proliferation in human carotid artery stenosis. J Biol Regul Homeost Agents 32: 506-516, 2018

36. Janczak D, Ziolkowski P, Szydelko T, Dorobisz T, Janczak D, Dorobisz K and Chabowski M: The presence of some cytokines and Chlamydia pneumoniae in the atherosclerotic carotid plaque in patients with carotid artery stenosis. Postepy Hig Med Dosw (Online) 69: 227-232, 2015.

37. Chen J, Cui L, Yuan J, Zhang Y and Sang H: Circular RNA WDR77 target FGF-2 to regulate vascular smooth muscle cells proliferation and migration by sponging miR-124. Biochem Biophys Res Commun 494: 126-132, 2017.

38. Zhou B, Ma R, Si W,Li S, Xu Y, Tu X and Wang Q: MicroRNA-503 targets FGF2 and VEGFA and inhibits tumor angiogenesis and growth. Cancer Lett 333: 159-169, 2013.

39. Kim J, Kang Y, Kojima Y, Lighthouse JK, Hu X, Aldred MA, McLean DL, Park H, Comhair SA, Greif DM, et al: An endothelial apelin-FGF link mediated by miR-424 and miR-503 is disrupted in pulmonary arterial hypertension. Nat Med 19: 74-82, 2013.

This work is licensed under a Creative Commons Attribution-NonCommercial-NoDerivatives 4.0 International (CC BY-NC-ND 4.0) License. 